\title{
A Compact Threshold Voltage Model for Gate Misalignment Effect of DG FD SOI nMOS Devices Considering Fringing Electric Field Effects
}

\author{
Elvis C. Sun and James B. Kuo, Fellow, IEEE
}

\begin{abstract}
This paper reports an analysis of gate misalignment effect on the threshold voltage of double-gate ultrathin fully depleted silicon-on-insulator nMOS devices using a compact model considering the fringing electric field effect, biased at zero-bias $V_{\mathrm{DS}}$. Using the conformal mapping transformation approach, a closed-form compact model considering the fringing electric field effect in the nongate overlap region has been derived to provide an accurate prediction of the threshold voltage behavior as verified by the two-dimensional simulation results.
\end{abstract}

Index Terms-Device modeling, MOS, silicon-on-insulator (SOI).

\section{INTRODUCTION}

D OUBLE-GATE (DG), silicon-on-insulator (SOI) technology has been regarded as another mainstream technology for sub-100-nm CMOS very large-scale integration (VLSI) owing to its advantages in reduced second-order effects[1], [2]. Many aspects of DG SOI devices have been studied [3]-[6]. For DG SOI CMOS devices, the gate misalignment has a large effect on device performance [7]. Fig. 1 shows the two-dimensional (2-D) electric field contours in a DG SOI nMOS device with a gate oxide thickness of $70 \AA$, a thin film of $600 \AA$ doped with a p-type density of $4 \times 10^{17} \mathrm{~cm}^{-3}$, and a gate misalignment of $0.04 \mu \mathrm{m}$, biased at $V_{G}=V_{\mathrm{th}}$, and $V_{D}=50 \mathrm{mV}$. As shown in the figure, the fringing electric field from the right edge of the bottom gate to the thin film of the nongate overlap region is substantial, which could have a nonnegligible influence on device performance. Several models for DG SOI devices have been reported [8]-[10]. However, until no analytical model for the gate misalignment effect has been reported. In this paper, an analysis of the gate misalignment effect on the threshold voltage of DG ultrathin FD SOI nMOS devices is reported, using a compact model that considers the fringing field effect. It is shown that by using the conformal mapping transformation approach, such a closed-form model, which is reported for the first time, can provide an accurate predication of the threshold voltage behavior as verified by the 2-D simulation results. In the following sections, derivation of

Manuscript received June 18, 2003; revised November 24, 2003. This work was supported by the National Science Council of Taiwan, R.O.C. The review of this paper was arranged by Editor C.-Y. Lu.

E. C. Sun is currently serving his military obligation in the Taiwanese Defense Force.

J. B. Kuo is with the Department of Electrical Engineering, National Taiwan University, Taipei, 106-17 Taiwan, R.O.C. (e-mail: jbkuo@cc.ee.ntu.edu.tw).

Digital Object Identifier 10.1109/TED.2004.825108 the analytical model is described, followed by model verification and discussion.

\section{Model Derivation}

In this section, a compact zero-biased threshold voltage considering the fringing electric field effect is derived. In order to concentrate on the fringing electric field effects from gate misalignment, a DG SOI nMOS device with a channel length of $0.2 \mu \mathrm{m}$ and a gate misalignment $\mathrm{m}_{a}$ as shown in Fig. 2(a), biased at its threshold voltage $V_{G}=V_{\mathrm{th}}$ and $V_{D}=50 \mathrm{mV}$ for the zero-bias $V_{\mathrm{DS}}$ condition, is considered. Define the origin of the coordinates at the tangent to the left edge of the top gate on the back surface. The $Y$-axis is along the back surface in the drain direction. The $X$-axis is in the vertical direction tangent to the left edge of the top gate. In the thin film, 2-D Poisson's equation is

$$
\frac{\partial^{2} \Psi(x, y)}{\partial x^{2}}+\frac{\partial^{2} \Psi(x, y)}{\partial y^{2}}=\frac{q N_{A}}{\epsilon_{\mathrm{si}}}
$$

where $N_{A}$ is the p-type doping density of the thin film, $\epsilon_{\mathrm{si}}$ is silicon permittivity, and q is electronic charge. In order to simplify the analysis, polysilicon depletion effect [11], [12] and quantum effects [13], [14] are not considered. In the following derivation, the thin film is divided into (I) gate overlap region and (II) nongate overlap region.

\section{A. Gate Overlap Region (I)}

In the gate overlap region (I), a 2-D parabolic approximation [10] has been used to solve 2-D Poisson's equation

$$
\Psi_{1}(x, y)=P_{01}(y)+P_{11}(y) x+P_{21}(y) x^{2} .
$$

The boundary conditions for the gate overlap region (I) are that at top and bottom thin-film interfaces, the displacements are continuous

$$
\begin{aligned}
& -\left.\frac{\partial \Psi_{1}(x, y)}{\partial x}\right|_{x=0}=\frac{C_{\mathrm{ox} 2}}{\epsilon_{\mathrm{Si}}}\left[V_{G}+\phi_{f 2}-\Psi_{b 1}(y)\right] \\
& -\left.\frac{\partial \Psi_{1}(x, y)}{\partial x}\right|_{x=t_{i}}=\frac{C_{\mathrm{ox} 1}}{\epsilon_{\mathrm{si}}}\left[\Psi_{s 1}(y)-V_{G}-\phi_{f 1}\right]
\end{aligned}
$$

where $C_{\mathrm{ox} 1 / 2}$ is the unit-area top/bottom gate oxide capacitance, $V_{G}$ is the gate voltage, $\phi_{f 1 / 2}=(k T / q) \ln \left(N_{\text {poly }} / n_{i}\right)$ is the Fermi voltage of the top/bottom gate, $\Psi_{b 1}(y)$ is the back surface potential $\left[\Psi_{b 1}(y)=\Psi_{1}(0, y)\right]$, and $\Psi_{s 1}(y)$ is the front surface potential $\left[\Psi_{s 1}(y)=\Psi_{1}\left(t_{i}, y\right)\right]$. Solving 2-D Poisson's equation 


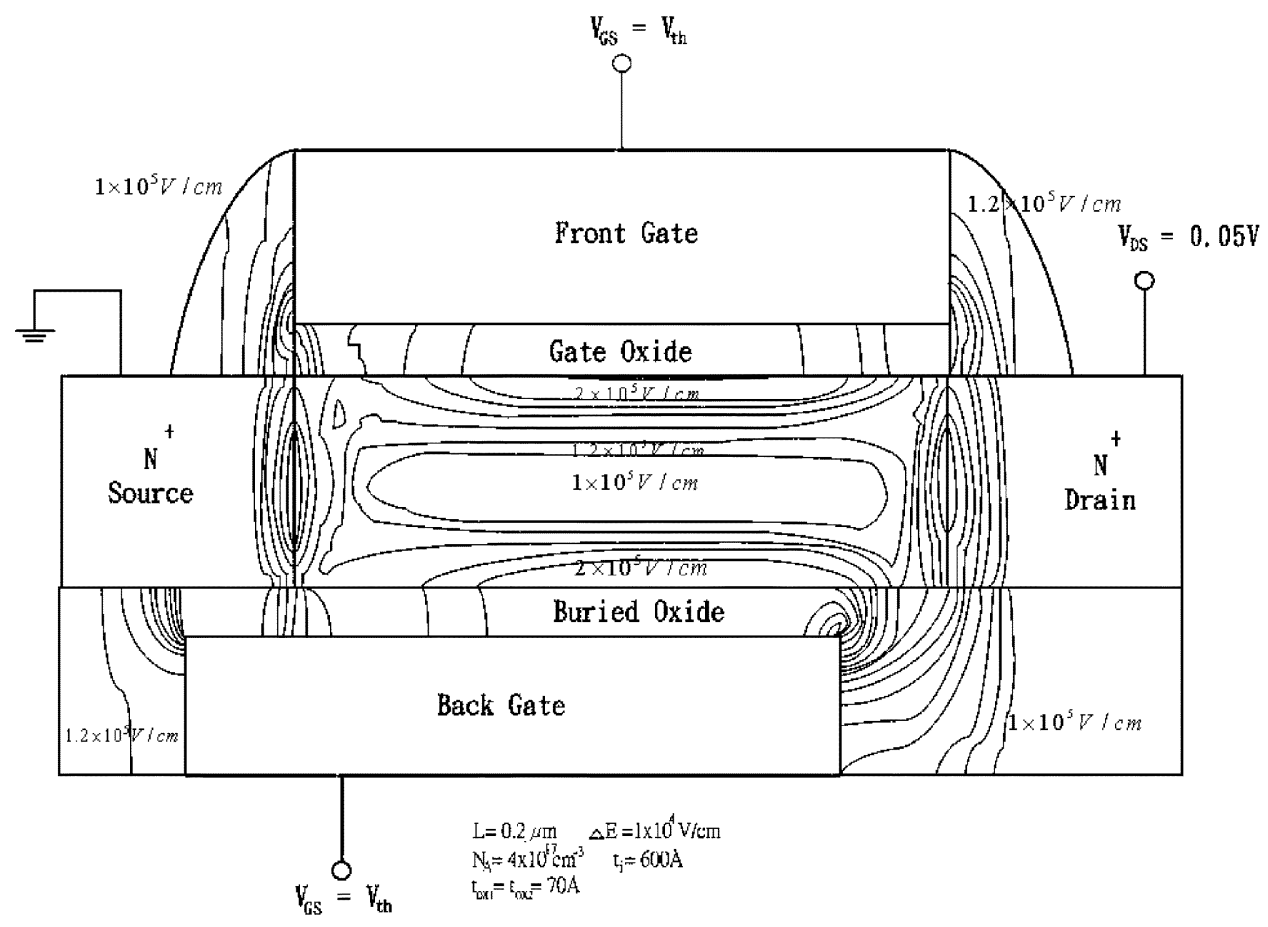

Fig. 1. Two-dimensional electric field contours in a DG SOI nMOS device with $\mathrm{n}^{+}$polysilicon top and bottom gates, a gate oxide of $70 \AA$, a thin film of $600 \AA$ doped with a p-type density of $4 \times 10^{17} \mathrm{~cm}^{-3}$, and a gate misalignment of $0.04 \mu \mathrm{m}$, biased at $V_{G}=V_{\mathrm{th}}$ and $V_{D}=50 \mathrm{mV}$. with the boundary conditions, the coefficients are shown in (4)
at the bottom of the page. In addition, a relationship between front and back surface potentials has been obtained

$\Psi_{s 1}(y)=\Psi_{b 1}(y)-\frac{C_{\mathrm{ox} 2}}{\epsilon_{\mathrm{si}}}\left[V_{G}+\phi_{f 2}-\Psi_{b 1}(y)\right] t_{i}+P_{21}(y) t_{i}^{2}$.

Since the thin film is very thin, we may assume that [10]

$$
\frac{\partial^{2} \Psi_{1}(y)}{\partial y^{2}} \cong \frac{1}{\kappa_{s}} \frac{\partial^{2} \Psi_{b 1}(y)}{\partial y^{2}}
$$

From (1), (2), (4) and (6), a differential equation in terms of the back surface potential is

$$
\begin{aligned}
& \frac{\partial^{2} \Psi_{b 1}(y)}{\partial y^{2}}-\kappa_{s} \frac{\left(1+\frac{C_{\mathrm{ox} 2}}{C_{s}}+\frac{C_{\mathrm{ox} 2}}{C_{\mathrm{ox} 1}}\right)}{\frac{1}{2} t_{i}^{2}\left(1+\frac{2 C_{s}}{C_{\mathrm{ox} 1}}\right)} \Psi_{b 1}(y) \\
& =\kappa_{s}\left[\frac{q N_{A}}{\epsilon_{\mathrm{si}}}-\frac{\left(1+\frac{C_{\mathrm{ox} 2}}{C_{s}}+\frac{C_{\mathrm{ox} 2}}{C_{\mathrm{ox} 1}}\right) V_{G}+\left(\frac{C_{\mathrm{ox} 2}}{C_{s}}+\frac{C_{\mathrm{ox} 2}}{C_{\mathrm{ox} 1}}\right) \phi_{f 2}+\phi_{f 1}}{\frac{1}{2} t_{i}^{2}\left(1+\frac{2 C_{s}}{C_{\mathrm{ox} 1}}\right)}\right] .
\end{aligned}
$$

Solving this differential equation, the back surface potential is

$$
\Psi_{b 1}(y)
$$$$
=C_{1} \exp \left(\sqrt{A_{0}} y\right)+C_{2} \exp \left(-\sqrt{A_{0}} y\right)-\frac{A_{1}}{A_{0}}
$$

$$
\begin{aligned}
& A_{0}=\kappa_{s}\left[\frac{\left(1+\frac{C_{\mathrm{ox} 2}}{C_{s}}+\frac{C_{\mathrm{ox} 2}}{C_{\mathrm{ox} 1}}\right)}{\frac{1}{2} t_{i}^{2}\left(1+\frac{2 C_{s}}{C_{\mathrm{ox} 1}}\right)}\right] \\
& A_{1}=\kappa_{s}\left[\frac{q N_{A}}{\epsilon_{\mathrm{Si}}}-\frac{\left(1+\frac{C_{\mathrm{ox} 2}}{C_{s}}+\frac{C_{\mathrm{ox} 2}}{C_{\mathrm{ox} 1}}\right) V_{G}+\left(\frac{C_{\mathrm{ox} 2}}{C_{s}}+\frac{C_{\mathrm{ox} 2}}{C_{\mathrm{ox} 1}}\right) \phi_{f 2}+\phi_{f 1}}{\frac{1}{2} t_{i}^{2}\left(1+\frac{2 C_{s}}{C_{\mathrm{ox} 1}}\right)}\right] .
\end{aligned}
$$

\section{B. Non-Gate Overlap Region (II)}

In the nongate overlap region (II), the electrostatic potential is approximated as

$$
\Psi_{2}(x, y)=P_{02}(y)+P_{12}(y) x+P_{22}(y) x^{2}
$$

$$
\begin{aligned}
& P_{01}(y)=\Psi_{b 1}(y) \\
& P_{11}(y)=-\frac{C_{\mathrm{ox} 2}}{\epsilon_{\mathrm{si}}}\left[V_{G}+\phi_{f 2}-\Psi_{b 1}(y)\right] \\
& P_{21}(y)=\frac{-\left[1+\frac{C_{\mathrm{ox} 2}}{C_{s}}+\frac{C_{\mathrm{ox} 2}}{C_{\mathrm{ox} 1}}\right] \Psi_{b 1}(y)+\left[1+\frac{C_{\mathrm{ox} 2}}{C_{s}}+\frac{C_{\mathrm{ox} 2}}{C_{\mathrm{ox} 1}}\right] V_{G}+\left[\frac{C_{\mathrm{ox} 2}}{C_{s}}+\frac{C_{\mathrm{ox} 2}}{C_{\mathrm{ox} 1}}\right] \phi_{f 2}+\phi_{f 1}}{t_{i}^{2}\left(1+\frac{2 C_{s}}{C_{\mathrm{ox} 1}}\right)} .
\end{aligned}
$$




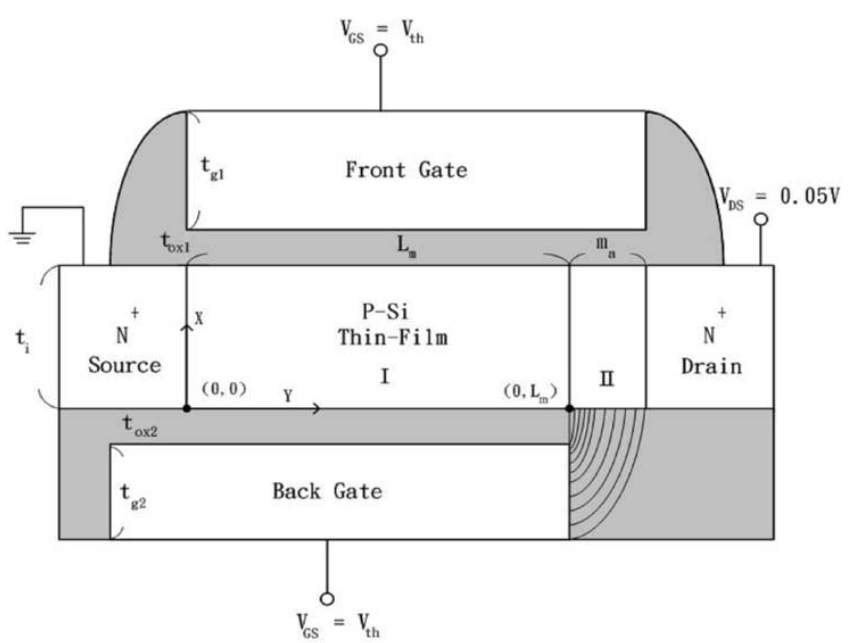

(a)
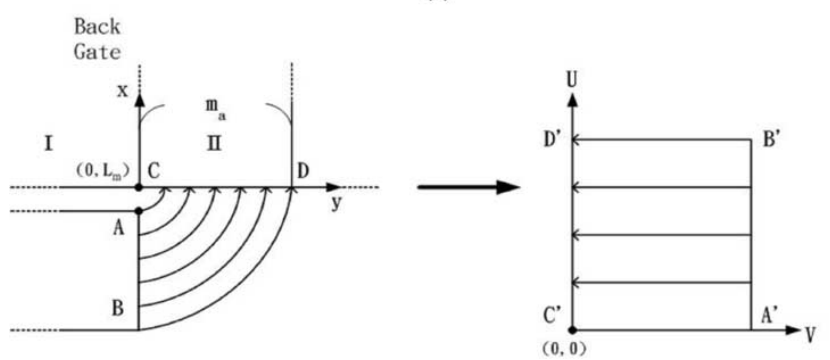

(b)

Fig. 2. (a) Cross section of the DG SOI nMOS device with a gate misalignment and (b) the boundary of the sidewall oxide region next to the right edge of the bottom gate before and after conformal mapping for model derivation.

The boundary conditions at top and bottom interfaces become

$$
\begin{aligned}
& -\left.\frac{\partial \Psi_{2}(x, y)}{\partial x}\right|_{x=0}=\frac{\epsilon_{\mathrm{ox}}}{\epsilon_{\mathrm{si}} t(y)}\left[V_{G}+\phi_{f 2}-\Psi_{b 2}(y)\right] \\
& -\left.\frac{\partial \Psi_{2}(x, y)}{\partial x}\right|_{x=t i}=\frac{C_{\mathrm{ox} 1}}{\epsilon_{\mathrm{si}}}\left[\Psi_{s 2}(y)-V_{G}-\phi_{f 1}\right]
\end{aligned}
$$

where $\Psi_{b 2}(y)=\Psi_{2}(0, y)$ and $\Psi_{s 2}=\Psi_{2}\left(t_{i}, y\right)$ are back and front surface potentials, respectively. $t(y)$ is the distance from the right edge of the bottom gate to the back surface channel. Due to the fringing electric field, $t(y)$ is not a fixed value. From (9) and (10), the coefficients are shown in (11) at the bottom of the page. In addition, a relationship between front and back surface potential is

$\Psi_{s 2}(y)=\Psi_{b 2}(y)-\frac{\epsilon_{\mathrm{ox}}}{\epsilon_{\mathrm{si}} t(y)}\left[V_{G}+\phi_{f 2}-\Psi_{b 2}(y)\right] t_{i}+P_{22}(y) t_{i}^{2}$.
Using a similar approximation approach as for the gate overlap region $(\mathrm{I})-\left(\partial^{2} \Psi_{2}(y) / \partial y^{2}\right) \cong\left(1 / \kappa_{s}\right)\left(\partial^{2} \Psi_{b 2}(y) / \partial y^{2}\right)$, from (9) and (1), one obtains

$$
\frac{\partial^{2} \Psi_{b 2}(y)}{\partial y^{2}}=\kappa_{s}\left[\frac{\partial^{2} \Psi_{2}(y)}{\partial y^{2}}\right]=\kappa_{s}\left[\frac{q N_{A}}{\epsilon_{\mathrm{si}}}-2 P_{22}(y)\right]
$$

which is a differential equation in terms of the back surface potential. Due to the fringing electric field from the right edge of the bottom gate via the oxide to the thin-film interface, the above equation is difficult to calculate. In order to simplify the analysis, a conformal mapping transformation technique [15] has been used to transform the original $x \vec{X}+y \vec{Y}$ space in terms of the $\vec{X}$ and $\vec{Y}$ axes as shown in Fig. 2 to the $u \vec{U}+v \vec{V}$ space in terms of $\vec{U}$ and $\vec{V}$ axes based on the following transfer function:

$$
\begin{aligned}
\left(y-L_{m}\right) \vec{Y}+n x \vec{X} & =k \sinh (u \vec{U}+v \vec{V}) \\
L_{m} & =L_{c}-m_{a} \\
n & =\frac{m_{a}}{t_{\mathrm{ox} 2} \sinh \left[\cosh ^{-1}\left(\frac{t_{\mathrm{ox} 2}+t_{g 2}}{t_{\mathrm{ox} 2}}\right)\right]} \\
k & =\frac{m_{a}}{\sinh \left[\cosh ^{-1}\left(\frac{t_{\mathrm{ox} 2}+t_{g 2}}{t_{\mathrm{ox} 2}}\right)\right]} .
\end{aligned}
$$

From the above formula, ABCD in the $x \vec{X}+y \vec{Y}$ coordinates is transformed into A'B'C'D' in the $u \vec{U}+v \vec{V}$ coordinates. As a result, the arc-shaped electric field contour in the oxide next to the right edge of the bottom gate in the $x \vec{X}+y \vec{Y}$ coordinates has become straight-line-shaped in the $u \vec{U}+v \vec{V}$ coordinates. Based on the transformation, the distance between the bottom gate electrode and the bottom thin-film/oxide interface $t(y)$, which is not a fixed value, has been transformed into $m \pi / 2$, which is the distance between points $\mathrm{A}^{\prime}$ and $\mathrm{C}^{\prime}$ in the new coordinates under the condition $\sinh (m \pi / 2)=1$. Therefore, after conformal mapping transformation $(t(y) \rightarrow m \pi / 2$, $\Psi_{b 2}(y) \rightarrow \Psi_{v}(v), y-L_{m}=k \sinh v$ ), a differential equation in terms of back surface potential $\Psi_{v}$ in the $u \vec{U}+v \vec{V}$ coordinates has been obtained

$$
\begin{aligned}
\frac{\partial^{2} \Psi_{v}(v)}{\partial y^{2}} & =\kappa_{s}\left[\frac{q N_{A}}{\epsilon_{\mathrm{si}}}-2 \alpha_{0} \Psi_{v}(v)-2 \beta_{0}\right] \\
\alpha_{0} & =-\frac{\left(1+\frac{2 t_{\mathrm{ox} 1}}{m \pi}+\frac{2 \epsilon_{\mathrm{ox}}}{m \pi C_{s}}\right)}{t_{i}^{2}\left(1+\frac{2 C_{s}}{C_{\mathrm{ox} 1}}\right)} \\
\beta_{0} & =\frac{\left[1+\frac{2 t_{\mathrm{ox} 1}}{m \pi}+\frac{2 \epsilon_{\mathrm{ox}}}{m \pi C_{s}}\right] V_{G}+\left[\frac{2 t_{\mathrm{ox} 1}}{m \pi}+\frac{2 \epsilon_{\mathrm{ox}}}{m \pi C_{s}}\right] \phi_{f 2}+\phi_{f 1}}{t_{i}^{2}\left(1+\frac{2 C_{s}}{C_{\mathrm{ox} 1}}\right)} .
\end{aligned}
$$

$$
\begin{aligned}
& P_{02}(y)=\Psi_{b 2}(y) \\
& P_{12}(y)=-\frac{\epsilon_{\mathrm{ox}}}{\epsilon_{\mathrm{Si}} t(y)}\left[V_{G}+\phi_{f 2}-\Psi_{b 2}(y)\right] \\
& P_{22}(y)=\frac{-\left[1+\frac{t_{\mathrm{ox} 1}}{t(y)}+\frac{\epsilon_{\mathrm{ox}}}{t(y) C_{s}}\right] \Psi_{b 2}(y)+\left[1+\frac{t_{\mathrm{ox} 1}}{t(y)}+\frac{\epsilon_{\mathrm{ox}}}{t(y) C_{s}}\right] V_{G}+\left[\frac{t_{\mathrm{ox} 1}}{t(y)}+\frac{\epsilon_{\mathrm{ox}}}{t(y) C_{s}}\right] \phi_{f 2}+\phi_{f 1}}{t_{i}^{2}\left(1+\frac{2 C_{s}}{C_{\mathrm{ox} 1}}\right)} .
\end{aligned}
$$


In addition, after transformation, the coefficients become

$$
\begin{aligned}
& P_{02}(v)=\Psi_{v}(v) \\
& P_{12}(v)=-\frac{2 \epsilon_{\mathrm{ox}}}{m \pi \epsilon_{\mathrm{si}}}\left[V_{G}+\phi_{f 2}-\Psi_{v}(v)\right] \\
& P_{22}(v)=\alpha_{0} \Psi_{v}(v)+\beta_{0} .
\end{aligned}
$$

Since

$$
\begin{aligned}
\frac{\partial^{2} \Psi_{v}(v)}{\partial y^{2}} & =\frac{\partial}{\partial y}\left(\frac{\partial v}{\partial y} \frac{\partial \Psi_{v}(v)}{\partial v}\right) \\
& =\left(\frac{\partial v}{\partial y}\right)^{2} \frac{\partial^{2} \Psi_{v}(v)}{\partial v^{2}}+\left(\frac{\partial^{2} v}{\partial y^{2}}\right) \frac{\partial \Psi_{v}(v)}{\partial v}
\end{aligned}
$$

the differential (15) could be rewritten as

$$
\begin{array}{r}
\frac{\partial^{2} \Psi_{v}(v)}{\partial v^{2}}-\tanh v \frac{\partial \Psi_{v}(v)}{\partial v}+2 \alpha_{0} \kappa_{s} k^{2} \cosh ^{2} v \Psi_{v}(v) \\
=\kappa_{s} k^{2} \cosh ^{2} v\left[\frac{q N_{A}}{\epsilon_{\mathrm{si}}}-2 \beta_{0}\right] .
\end{array}
$$

Equation (17) is a nonlinear differential equation, which is difficult to solve. In order to simplify the situation, one may assume that $a=\sinh v$ and thus, $\left(\partial \Psi_{v} / \partial v\right)=\left(\partial \Psi_{v} / \partial a\right) \cosh v$, and

$$
\frac{\partial^{2} \Psi_{v}}{\partial v^{2}}=\left(\frac{\partial^{2} \Psi_{v}}{\partial a^{2}}\right) \cosh ^{2} v+\left(\frac{\partial \Psi_{v}}{\partial a}\right) \sinh v .
$$

Via this, (17) is further simplified as a linear differential equation

$$
\begin{aligned}
\frac{\partial^{2} \Psi_{v}}{\partial a^{2}}+B_{0} \Psi_{v} & =B_{1} \\
B_{0} & =2 \kappa_{s} k^{2} \alpha_{0} \\
B_{1} & =\kappa_{s} k^{2}\left[\frac{q N_{A}}{\epsilon_{\mathrm{si}}}-2 \beta_{0}\right] .
\end{aligned}
$$

Solving (18), one obtains the back surface potential in the $u \vec{U}+v \vec{V}$ coordinates as

$$
\begin{aligned}
\Psi_{v} & =g_{1} \exp (\gamma a)+g_{2} \exp (-\gamma a)+\frac{B_{1}}{B_{0}} \\
\gamma & =\sqrt{-B_{0}} .
\end{aligned}
$$

Using $\sinh v=y-L_{m} / k$ to transform back to the $x \vec{X}+y \vec{Y}$ coordinates, the back surface potential is

$$
\Psi_{b 2}(y)=g_{1} \exp \left[\frac{\gamma}{k}\left(y-L_{m}\right)\right]+g_{2} \exp \left[-\frac{\gamma}{k}\left(y-L_{m}\right)\right]+\frac{B_{1}}{B_{0}} .
$$

\section{Boundary Conditions}

In this subsection, boundary conditions for back and front surface potentials are described.

1) Back Surface Potentials $\left(\psi_{b 1}(y), \psi_{b 2}(y)\right)$ : For the back surface potentials in the gate overlap and the nongate overlap regions $\left(\Psi_{b 1}(y), \Psi_{b 2}(y)\right)$, the boundary conditions are that at the left $(y=0)$ and the $\operatorname{right}\left(y=L_{c}\right)$ sides, they are determined by the source and the drain potentials

$$
\begin{gathered}
\Psi_{b 1}(0)=\phi_{f n}+V_{S} \\
\Psi_{b 2}\left(L_{c}\right)=\phi_{f n}+V_{D} .
\end{gathered}
$$

where $\phi_{f n}=(k T / q) \ln \left(N_{D} / n_{i}\right), N_{D}$ is the source/drain doping density. At the boundary $\left(y=L_{m}\right)$ between the gate overlap and the nongate overlap regions, the potential and the electric field are continuous. Thus

$$
\begin{aligned}
\Psi_{b 1}\left(L_{m}\right) & =\Psi_{b 2}\left(L_{m}\right) \\
\left.\frac{\partial \Psi_{b 1}}{\partial y}\right|_{y=L_{m}} & =\left.\frac{\partial \Psi_{b 2}}{\partial y}\right|_{y=L_{m}} .
\end{aligned}
$$

From $\Psi_{b 1}(y)$, (8), and $\Psi_{b 2}(y)$, (20), with four boundary conditions, (21) and (22), the coefficients are shown in (23) at the bottom of the page.

2) Front Surface Potential $\left(\psi_{s 1}(y), \psi_{s 2}(y)\right)$ : From (11) and (12), a relationship between the front surface potential $\Psi_{s 2}$ and the back surface potential $\Psi_{b 2}$ in the nongate overlap region could be found as

$\Psi_{s 2}(y)=\frac{\sigma_{2}\left[\frac{2 C_{s}}{C_{\mathrm{ox} 1}}+\frac{t_{\mathrm{ox} 1}}{t(y)}\right] \Psi_{b 2}(y)+\left[1-\frac{t_{\mathrm{ox} 1}}{t(y)}\right] V_{G}-\left[\frac{t_{\mathrm{ox} 1}}{t(y)}\right] \phi_{f 2}+\phi_{f 1}}{1+\frac{2 C_{s}}{C_{\mathrm{ox} 1}}}$

where $\sigma_{2}=0.51+4 m_{a} \times 10^{4}$ is an empirical correction factor to account for the nonsymmetric structure in the nongate overlap region. Note that $m_{a}$ is the gate misalignment in centimeter.

$$
\begin{aligned}
& C_{1}=\frac{\left(\phi_{f n}+V_{S}+\frac{A_{1}}{A_{0}}\right) \exp \left(-\sqrt{A_{0}} L_{m}\right)\left[\cosh \left(\frac{\gamma}{k} m_{a}\right)-\sinh \left(\frac{\gamma}{k} m_{a}\right) \frac{\sqrt{A_{0}} k}{\gamma}\right]-\phi_{f n}-V_{D}+\frac{B_{1}}{B_{0}}-\left(\frac{A_{1}}{A_{0}}+\frac{B_{1}}{B_{0}}\right) \cosh \left(\frac{\gamma}{k} m_{a}\right)}{-2\left[\sinh \left(\sqrt{A_{0}} L_{m}\right) \cosh \left(\frac{\gamma}{k} m_{a}\right)+\cosh \left(\sqrt{A_{0}} L_{m}\right) \sinh \left(\frac{\gamma}{k} m_{a}\right) \frac{\sqrt{A_{0}} k}{\gamma}\right]} \\
& C_{2}=\frac{\left(\phi_{f n}+V_{S}+\frac{A_{1}}{A_{0}}\right) \exp \left(\sqrt{A_{0}} L_{m}\right)\left[\cosh \left(\frac{\gamma}{k} m_{a}\right)+\sinh \left(\frac{\gamma}{k} m_{a}\right) \frac{\sqrt{A_{0}} k}{\gamma}\right]-\phi_{f n}-V_{D}+\frac{B_{1}}{B_{0}}-\left(\frac{A_{1}}{A_{0}}+\frac{B_{1}}{B_{0}}\right) \cosh \left(\frac{\gamma}{k} m_{a}\right)}{2\left[\sinh \left(\sqrt{A_{0}} L_{m}\right) \cosh \left(\frac{\gamma}{k} m_{a}\right)+\cosh \left(\sqrt{A_{0}} L_{m}\right) \sinh \left(\frac{\gamma}{k} m_{a}\right) \frac{\sqrt{A_{0}} k}{\gamma}\right]} \\
& g_{1}=\frac{\left(\phi_{f n}+V_{D}-\frac{B_{1}}{B_{0}}\right)\left[\cosh \left(\sqrt{A_{0}} L_{m}\right)+\sinh \left(\sqrt{A_{0}} L_{m}\right) \frac{\gamma}{k \sqrt{A_{0}}}\right]+\left[\left(\frac{A_{1}}{A_{0}}+\frac{B_{1}}{B_{0}}\right) \cosh \left(\sqrt{A_{0}} L_{m}\right)-\phi_{f n}-V_{S}-\frac{A_{1}}{A_{0}}\right] \exp \left(-\frac{\gamma}{k} m_{a}\right)}{2\left[\cosh \left(\sqrt{A_{0}} L_{m}\right) \sinh \left(\frac{\gamma}{k} m_{a}\right)+\sinh \left(\sqrt{A_{0}} L_{m}\right) \cosh \left(\frac{\gamma}{k} m_{a}\right) \frac{\gamma}{k \sqrt{A_{0}}}\right]} \\
& g_{2}=\frac{\left(\phi_{f n}+V_{D}-\frac{B_{1}}{B_{0}}\right)\left[\cosh \left(\sqrt{A_{0}} L_{m}\right)-\sinh \left(\sqrt{A_{0}} L_{m}\right) \frac{\gamma}{k \sqrt{A_{0}}}\right]-\left[\left(\frac{A_{1}}{A_{0}}+\frac{B_{1}}{B_{0}}\right) \cosh \left(\sqrt{A_{0}} L_{m}\right)+\phi_{f n}+V_{S}+\frac{A_{1}}{A_{0}}\right] \exp \left(\frac{\gamma}{k} m_{a}\right)}{-2\left[\cosh \left(\sqrt{A_{0}} L_{m}\right) \sinh \left(\frac{\gamma}{k} m_{a}\right)+\sinh \left(\sqrt{A_{0}} L_{m}\right) \cosh \left(\frac{\gamma}{k} m_{a}\right) \frac{\gamma}{k \sqrt{A_{0}}}\right]}
\end{aligned}
$$


From (4) and (5), a relationship between the front surface potential $\Psi_{s 1}$ and the back surface potential $\Psi_{b 1}$ in the gate overlap region has been found as

$\Psi_{s 1}(y)=\frac{\sigma_{1}\left[\frac{2 C_{s}}{C_{\mathrm{ox} 1}}+\frac{C_{\mathrm{ox} 2}}{C_{\mathrm{ox} 1}}\right] \Psi_{b 1}(y)+\left[1-\frac{C_{\mathrm{ox} 2}}{C_{\mathrm{ox} 1}}\right] V_{G}-\left[\frac{C_{\mathrm{ox} 2}}{C_{\mathrm{ox} 1}}\right] \phi_{f 2}+\phi_{f 1}}{1+\frac{2 C_{s}}{C_{\mathrm{ox} 1}}}$

where $\sigma_{1}=0.99-2 m_{a} \times 10^{4}$ is an empirical correction factor.

From (20) and (24) and (8) and (25), the front surface potentials are

$$
\begin{aligned}
\Psi_{s 1}(y) & =C_{3} \exp \left(\sqrt{A_{0}} y\right)+C_{4} \exp \left(-\sqrt{A_{0}} y\right)-D_{1} \\
\Psi_{s 2}(y) & =g_{3} \exp \left[\frac{\gamma}{k}\left(y-L_{m}\right)\right]+g_{4} \exp \left[-\frac{\gamma}{k}\left(y-L_{m}\right)\right]+D_{2} \\
D_{1} & =\frac{\sigma_{1}\left[\frac{2 C_{s}}{C_{\mathrm{ox} 1}}+\frac{C_{\mathrm{ox} 2}}{C_{\mathrm{ox} 1}}\right] \frac{A_{1}}{A_{0}}+\left[\frac{C_{\mathrm{ox} 2}}{C_{\mathrm{ox} 1}}-1\right] V_{G}+\left[\frac{C_{\mathrm{ox} 2}}{C_{\mathrm{ox} 1}}\right] \phi_{f 2}-\phi_{f 1}}{1+\frac{2 C_{s}}{C_{\mathrm{ox} 1}}} \\
D_{2} & =\frac{\sigma_{2}\left[\frac{2 C_{s}}{C_{\mathrm{ox} 1}}+\frac{t_{\mathrm{ox} 1}}{t(y)}\right] \frac{B_{1}}{B_{0}}+\left[1-\frac{t_{\mathrm{ox} 1}}{t(y)}\right] V_{G}-\left[\frac{t_{\mathrm{ox} 1}}{t(y)}\right] \phi_{f 2}+\phi_{f 1}}{1+\frac{2 C_{s}}{C_{\mathrm{ox} 1}}} .
\end{aligned}
$$

For the front surface potentials in the gate overlap and the nongate overlap regions $\left(\Psi_{s 1}(y), \Psi_{s 2}(y)\right)$, the boundary conditions are that at the left $(y=0)$ and the $\operatorname{right}\left(y=L_{c}\right)$ sides, they are determined by the source and the drain potentials:

$$
\begin{gathered}
\Psi_{s 1}(0)=\phi_{f n}+V_{S} \\
\Psi_{s 2}\left(L_{c}\right)=\phi_{f n}+V_{D} .
\end{gathered}
$$

At the boundary $\left(y=L_{m}\right.$ ) between the gate overlap and the nongate overlap regions, the potential and the electric field are continuous. Thus

$$
\begin{aligned}
\Psi_{s 1}\left(L_{m}\right) & =\Psi_{s 2}\left(L_{m}\right) \\
\left.\frac{\partial \Psi_{s 1}}{\partial y}\right|_{y=L_{m}} & =\left.\frac{\partial \Psi_{s 2}}{\partial y}\right|_{y=L_{m}} .
\end{aligned}
$$

Using the boundary conditions (26)-(28), the coefficients in the front surface potentials in the gate overlap and the nongate overlap regions [(26)] are shown in (29) at the bottom of the page.
3) Threshold Voltage: The threshold voltage of the device is defined as the gate voltage when the sum of the electron densities at the locations in the front and the back surface channels with their respective minimum potentials equal to the doping density of the thin film

$$
N_{A}=n_{i} \exp \left(\frac{\Psi_{s \min }}{V_{T}}\right)+n_{i} \exp \left(\frac{\Psi_{b \min }}{V_{T}}\right) .
$$

where $\Psi_{s \text { min }}$ and $\Psi_{b \text { min }}$ are the minimum front and back surface potentials, respectively. Note that (30) is not applicable for the thin film with a zero or very light doping density, where $N_{A}$ should be replaced by $J / q \mu_{n} E$ ( $J$ is the predefined current density, $\mu_{n}$ is the electron mobility, and $E$ is the lateral electric field). In the gate overlap region, from $\partial \Psi_{b 1} / \partial y=0$, the minimum back surface potential is $\Psi_{b 1 \text { min }}=2 \sqrt{C_{1} C_{2}}-A_{1} / A_{0}$ at $y_{b 1 \min }=-\left(1 / 2 \sqrt{A_{0}}\right) \ln \left(C_{1} / C_{2}\right)$. In the nongate overlap region, from $\partial \Psi_{b 2} / \partial y=0$, the minimum back surface potential is $\Psi_{b 2 \min }=2 \sqrt{g_{1} g_{2}}+B_{1} / B_{0}$ at $y_{b 2 \min }=L_{m}$ $(k / 2 \gamma) \ln \left(g_{1} / g_{2}\right)$. From $\Psi_{b 1 \text { min }}$ and $\Psi_{b 2 \text { min }}, \Psi_{b \text { min }}$ could be determined. Similarly, the minimum front surface potential in the gate overlap region is $\Psi_{s 1 \text { min }}=2 \sqrt{C_{3} C_{4}}-D_{1}, y_{s 1 \text { min }}=$ $-\left(1 / 2 \sqrt{A_{0}}\right) \ln \left(C_{3} / C_{4}\right)$. The minimum front surface potential in the nongate overlap region is: $\Psi_{s 2 \text { min }}=2 \sqrt{g_{3} g_{4}}+D_{2}$, $y_{s 2 \min }=L_{m}-(k / 2 \gamma) \ln \left(g_{3} / g_{4}\right)$. From $\Psi_{s 1 \min }$ and $\Psi_{s 2 \min }$, $\Psi_{s \text { min }}$ could be found. As shown in Fig. 5(a), the minimum front surface potential ( $\left.\Psi_{s \text { min }}\right)$ is higher than the minimum back surface potential $\left(\Psi_{b \min }\right)$. Therefore, the second term in (30) can be neglected. If the minimum front surface potential occurs in the nongate overlap region, $\Psi_{s \min }=\Psi_{s 2 \min }$ and $y_{s 2 \min }=$ $L_{m}-(k / 2 \gamma) \ln \left(g_{3} / g_{4}\right)>L_{m}$, hence $g_{3}<g_{4}$, which can be reorganized as

$$
\begin{aligned}
\left(\phi_{f n}+V_{D}\right. & \left.-D_{2}\right) \cosh \left(\sqrt{A_{0}} L_{m}\right)+\left[\phi_{f n}+V_{s}+D_{1}\right. \\
& \left.-\left(D_{1}+D_{2}\right) \cosh \left(\sqrt{A_{0}} L_{m}\right)\right] \sinh \left(\frac{\gamma}{k} m_{a}\right)<0
\end{aligned}
$$

using (29). Under this situation, from (30), the threshold voltage is

$V_{\mathrm{th}}=\frac{-Z_{2}-\sqrt{Z_{2}^{2}-4 Z_{1} Z_{3}}}{2 Z_{1}}$

$$
\begin{aligned}
& C_{3}= \frac{\left(\phi_{f n}+V_{S}+D_{1}\right) \exp \left(-\sqrt{A_{0}} L_{m}\right)\left[\cosh \left(\frac{\gamma}{k} m_{a}\right)-\sinh \left(\frac{\gamma}{k} m_{a}\right) \frac{\sqrt{A_{0}} k}{\gamma}\right]-\phi_{f n}-V_{D}+D_{2}-\left(D_{1}+D_{2}\right) \cosh \left(\frac{\gamma}{k} m_{a}\right)}{-2\left[\sinh \left(\sqrt{A_{0}} L_{m}\right) \cosh \left(\frac{\gamma}{k} m_{a}\right)+\cosh \left(\sqrt{A_{0}} L_{m}\right) \sinh \left(\frac{\gamma}{k} m_{a}\right) \frac{\sqrt{A_{0}} k}{\gamma}\right]} \\
& C_{4}=\frac{\left(\phi_{f n}+V_{S}+D_{1}\right) \exp \left(\sqrt{A_{0}} L_{m}\right)\left[\cosh \left(\frac{\gamma}{k} m_{a}\right)+\sinh \left(\frac{\gamma}{k} m_{a}\right) \frac{\sqrt{A_{0}} k}{\gamma}\right]-\phi_{f n}-V_{D}+D_{2}-\left(D_{1}+D_{2}\right) \cosh \left(\frac{\gamma}{k} m_{a}\right)}{2\left[\sinh \left(\sqrt{A_{0}} L_{m}\right) \cosh \left(\frac{\gamma}{k} m_{a}\right)+\cosh \left(\sqrt{A_{0}} L_{m}\right) \sinh \left(\frac{\gamma}{k} m_{a}\right) \frac{\sqrt{A_{0}} k}{\gamma}\right]} \\
& g_{3}=\frac{\left(\phi_{f n}+V_{D}-D_{2}\right)\left[\cosh \left(\sqrt{A_{0}} L_{m}\right)+\sinh \left(\sqrt{A_{0}} L_{m}\right) \frac{\gamma}{k \sqrt{A_{0}}}\right]+\left[\left(D_{1}+D_{2}\right) \cosh \left(\sqrt{A_{0}} L_{m}\right)-\phi_{f n}-V_{S}-D_{1}\right] \exp \left(-\frac{\gamma}{k} m_{a}\right)}{2\left[\cosh \left(\sqrt{A_{0}} L_{m}\right) \sinh \left(\frac{\gamma}{k} m_{a}\right)+\sinh \left(\sqrt{A_{0}} L_{m}\right) \cosh \left(\frac{\gamma}{k} m_{a}\right) \frac{\gamma}{k \sqrt{A_{0}}}\right]} \\
& g_{4}=\frac{\left(\phi_{f n}+V_{D}-D_{2}\right)\left[\cosh \left(\sqrt{A_{0}} L_{m}\right)-\sinh \left(\sqrt{A_{0}} L_{m}\right) \frac{\gamma}{k \sqrt{A_{0}}}\right]-\left[\left(D_{1}+D_{2}\right) \cosh \left(\sqrt{A_{0}} L_{m}\right)+\phi_{f n}+V_{S}+D_{1}\right] \exp \left(\frac{\gamma}{k} m_{a}\right)}{-2\left[\cosh \left(\sqrt{A_{0}} L_{m}\right) \sinh \left(\frac{\gamma}{k} m_{a}\right)+\sinh \left(\sqrt{A_{0}} L_{m}\right) \cosh \left(\frac{\gamma}{k} m_{a}\right) \frac{\gamma}{k \sqrt{A_{0}}}\right]} .
\end{aligned}
$$




$$
\begin{aligned}
Z_{1}= & E_{3}^{2}+\frac{d_{2} d_{4}}{p_{0}^{2}} \\
Z_{2}= & 2 E_{3}\left(E_{4}-\frac{k T}{q} \ln \left(\frac{N_{A}}{n_{i}}\right)\right)+\frac{d_{1} d_{4}+d_{2} d_{3}}{p_{0}^{2}} \\
Z_{3}= & \left(E_{4}-\frac{k T}{q} \ln \left(\frac{N_{A}}{n_{i}}\right)\right)^{2}+\frac{d_{1} d_{3}}{p_{0}^{2}} \\
d_{1}= & \left(\phi_{f n}+V_{D^{-}}-E_{4}\right)\left[\cosh \left(\sqrt{A_{0}} L_{m}\right)+\sinh \left(\sqrt{A_{0}} L_{m}\right) \frac{\gamma}{k \sqrt{A_{0}}}\right] \\
& +\left[-\phi_{f n}-V_{S}-E_{2}+\left(E_{2}+E_{4}\right) \cosh \left(\sqrt{A_{0}} L_{m}\right)\right] \exp \left(-\frac{\gamma}{k} m_{a}\right) \\
d_{2}= & -E_{3}\left[\cosh \left(\sqrt{A_{0}} L_{m}\right)+\sinh \left(\sqrt{A_{0}} L_{m}\right) \frac{\gamma}{k \sqrt{A_{0}}}\right] \\
& +\left[-E_{1}+\left(E_{1}+E_{3}\right) \cosh \left(\sqrt{A_{0}} L_{m}\right)\right] \exp \left(-\frac{\gamma}{k} m_{a}\right) \\
d_{3}= & \left(\phi_{f n}+V_{D^{-}}-E_{4}\right)\left[\cosh \left(\sqrt{A_{0}} L_{m}\right)-\sinh \left(\sqrt{A_{0}} L_{m}\right) \frac{\gamma}{k \sqrt{A_{0}}}\right] \\
& +\left[-\phi_{f n}-V_{S}-E_{2}+\left(E_{2}+E_{4}\right) \cosh \left(\sqrt{A_{0}} L_{m}\right)\right] \exp \left(\frac{\gamma}{k} m_{a}\right) \\
d_{4}= & -E_{3}\left[\cosh \left(\sqrt{A_{0}} L_{m}\right)-\sinh \left(\sqrt{A_{0}} L_{m}\right) \frac{\gamma}{k \sqrt{A_{0}}}\right] \\
& +\left[-E_{1}+\left(E_{1}+E_{3}\right) \cosh \left(\sqrt{A_{0}} L_{m}\right)\right] \exp \left(\frac{\gamma}{k} m_{a}\right) \\
& (33) \\
&
\end{aligned}
$$$$
E_{1}=\frac{C_{\mathrm{ox} 2}-C_{\mathrm{ox} 1}-\sigma_{1}\left(2 C_{s}+C_{\mathrm{ox} 2}\right)}{C_{\mathrm{ox} 1}+2 C_{s}}
$$$$
E_{2}=\frac{C_{\mathrm{ox} 2} \phi_{f 2}-C_{\mathrm{ox} 1} \phi_{f 1}+\sigma_{1}\left(2 C_{s}+C_{\mathrm{ox} 2}\right) A_{2}}{C_{\mathrm{ox} 1}+2 C_{s}}
$$$$
E_{3}=\frac{C_{\mathrm{ox} 1}-\frac{\epsilon_{\mathrm{ox}}}{t(y)}+\sigma_{2}\left(2 C_{s}+\frac{\epsilon_{\mathrm{ox}}}{t(y)}\right)}{C_{\mathrm{ox} 1}+2 C_{s}}
$$$$
E_{4}=\frac{C_{\mathrm{ox} 1} \phi_{f 1}-\frac{\epsilon_{\mathrm{ox}}}{t(y)} \phi_{f 2}+\sigma_{2}\left(2 C_{s}+\frac{\epsilon_{\mathrm{ox}}}{t(y)}\right) B_{2}}{C_{\mathrm{ox} 1}+2 C_{s}}
$$$$
A_{2}=\frac{\frac{1}{2} q N_{A} t_{i}^{2}\left(1+\frac{2 C_{s}}{C_{\mathrm{ox} 1}}\right)-\epsilon_{\mathrm{si}}\left(\frac{C_{\mathrm{ox} 2}}{C_{s}}+\frac{C_{\mathrm{ox} 2}}{C_{\mathrm{ox} 1}}\right) \phi_{f 2}-\epsilon_{\mathrm{si}} \phi_{f 1}}{\epsilon_{\mathrm{si}}\left(1+\frac{C_{\mathrm{ox} 2}}{C_{s}}+\frac{C_{\mathrm{ox} 2}}{C_{\mathrm{ox} 1}}\right)}
$$$$
B_{2}=\frac{-q N_{A} t_{i}^{2}\left(1+\frac{2 C_{s}}{C_{\mathrm{ox} 1}}\right)+\epsilon_{\mathrm{si}}\left(\frac{2 t_{\mathrm{ox} 1}}{m \pi}+\frac{2 \epsilon_{\mathrm{ox}}}{m \pi C_{s}}\right) \phi_{f 2}+\epsilon_{\mathrm{si}} \phi_{f 1}}{\epsilon_{\mathrm{Si}}\left(1+\frac{2 t_{\mathrm{ox} 1}}{m \pi}+\frac{2 \epsilon_{\mathrm{ox}}}{m \pi C_{s}}\right)}
$$$$
\begin{aligned}
p_{0}= & \cosh \left(\sqrt{A_{0}} L_{m}\right) \sinh \left(\frac{\gamma}{k} m_{a}\right) \\
& +\sinh \left(\sqrt{A_{0}} L_{m}\right) \cosh \left(\frac{\gamma}{k} m_{a}\right) \frac{\gamma}{k \sqrt{A_{0}}} .
\end{aligned}
$$

$$
\begin{aligned}
& b_{4}=E_{1} \exp \left(\sqrt{A_{0}} L_{m}\right)\left[\cosh \left(\frac{\gamma}{k} m_{a}\right)+\sinh \left(\frac{\gamma}{k} m_{a}\right) \frac{\sqrt{A_{0}} k}{\gamma}\right] \\
& +E_{3}-\left(E_{1}+E_{3}\right) \cosh \left(\frac{\gamma}{k} m_{a}\right) \\
& f_{0}=\sinh \left(\sqrt{A_{0}} L_{m}\right) \cosh \left(\frac{\gamma}{k} m_{a}\right) \\
& +\cosh \left(\sqrt{A_{0}} L_{m}\right) \sinh \left(\frac{\gamma}{k} m_{a}\right) \frac{\sqrt{A_{0}} k}{\gamma} . \\
& X_{1}=E_{1}^{2}+\frac{b_{2} b_{4}}{f_{0}^{2}} \\
& X_{2}=2 E_{1}\left(E_{2}+\frac{k T}{q} \ln \left(\frac{N_{A}}{n_{i}}\right)\right)+\frac{b_{1} b_{4}+b_{2} b_{3}}{f_{0}^{2}} \\
& X_{3}=\left(E_{2}+\frac{k T}{q} \ln \left(\frac{N_{A}}{n_{i}}\right)\right)^{2}+\frac{b_{1} b_{3}}{f_{0}^{2}} \\
& b_{1}=\left(\phi_{f n}+V_{S}+E_{2}\right) \exp \left(-\sqrt{A_{0}} L_{m}\right) \\
& \cdot\left[\cosh \left(\frac{\gamma}{k} m_{a}\right)-\sinh \left(\frac{\gamma}{k} m_{a}\right) \frac{\sqrt{A_{0}} k}{\gamma}\right] \\
& -\phi_{f n}-V_{D}+E_{4}-\left(E_{2}+E_{4}\right) \cosh \left(\frac{\gamma}{k} m_{a}\right) \\
& b_{2}=E_{1} \exp \left(-\sqrt{A_{0}} L_{m}\right)\left[\cosh \left(\frac{\gamma}{k} m_{a}\right)-\sinh \left(\frac{\gamma}{k} m_{a}\right) \frac{\sqrt{A_{0}} k}{\gamma}\right] \\
& +E_{3}-\left(E_{1}+E_{3}\right) \cosh \left(\frac{\gamma}{k} m_{a}\right) \\
& b_{3}=\left(\phi_{f n}+V_{S}+E_{2}\right) \exp \left(\sqrt{A_{0}} L_{m}\right) \\
& \cdot\left[\cosh \left(\frac{\gamma}{k} m_{a}\right)+\sinh \left(\frac{\gamma}{k} m_{a}\right) \frac{\sqrt{A_{0}} k}{\gamma}\right] \\
& -\phi_{f n}-V_{D}+E_{4}-\left(E_{2}+E_{4}\right) \cosh \left(\frac{\gamma}{k} m_{a}\right)
\end{aligned}
$$

Note that the threshold voltage formula as shown in (31) and (37) is a function of the physical parameters of the DG SOI device as shown in related equations.

4) Without Considering the Fringing Effect: If the fringing electric field effect from the right edge of the bottom gate is not considered $\left(\partial \Psi_{2}(x, y) /\left.\partial x\right|_{x=0}=0\right)$, from (10), one obtains $P_{12}=0, P_{02}=\Psi_{b 2}$, and $P_{22}=$ $\left(-\Psi_{s 2}+V_{G}+\phi_{f 1}\right) /\left((1 / 2) t_{i}^{2}\left(1+2 C_{s} / C_{\mathrm{ox} 1}\right)\right)$. From (13), the differential equation in terms of the back surface potential is

$$
\frac{\partial^{2} \Psi_{b 2}}{\partial y^{2}}-\frac{2 \kappa_{s}}{t_{i}^{2}\left(1+\frac{2 C_{s}}{C_{\mathrm{ox} 1}}\right)} \Psi_{b 2}=\kappa_{s}\left[\frac{q N_{a}}{\epsilon_{\mathrm{si}}}-\frac{2\left(V_{G}+\phi_{f 1}\right)}{t_{i}^{2}\left(1+\frac{2 C_{s}}{C_{\mathrm{ox} 1}}\right)}\right] .
$$

On the other hand, when

$$
\begin{aligned}
\left(\phi_{f n}+V_{D}\right. & \left.-D_{2}\right) \cosh \left(\sqrt{A_{0}} L_{m}\right)+\left[\phi_{f n}+V_{s}+D_{1}\right. \\
& \left.-\left(D_{1}+D_{2}\right) \cosh \left(\sqrt{A_{0}} L_{m}\right)\right] \sinh \left(\frac{\gamma}{k} m_{a}\right)>0
\end{aligned}
$$

$\left(g_{3}>g_{4}\right)$, the minimum front surface potential occurs in the gate overlap region, which means $\Psi_{s \min }=\Psi_{s 1 \mathrm{~min}}$. Thus, the threshold voltage becomes

$V_{\mathrm{th}}=\frac{-X_{2}-\sqrt{X_{2}^{2}-4 X_{1} X_{3}}}{2 X_{1}}$ 


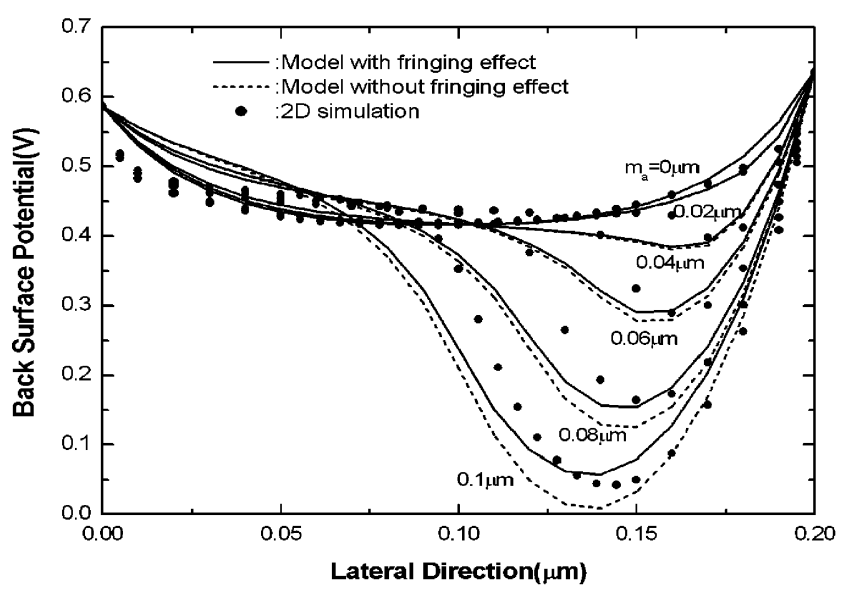

(a)

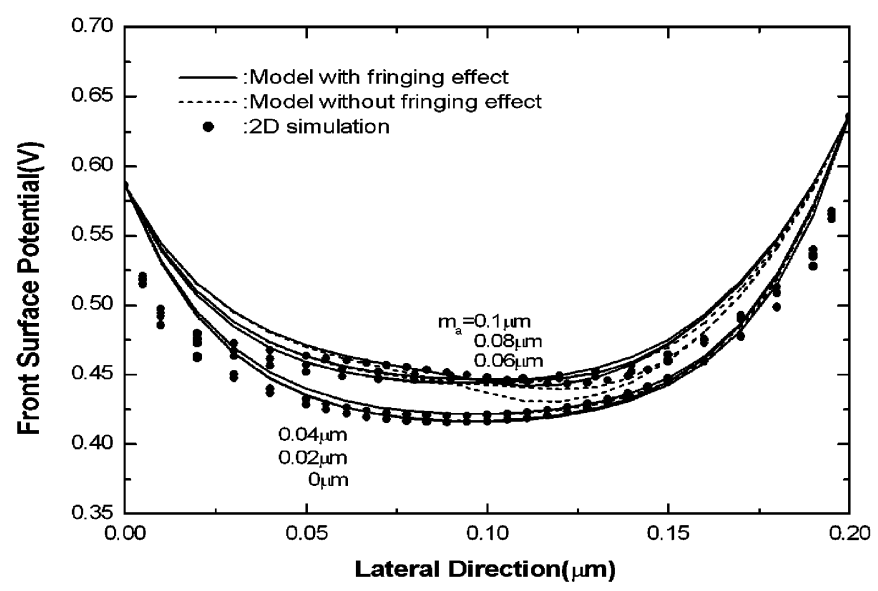

(b)

Fig. 3. (a) Back and (b) front surface potential distributions of the DG SOI nMOS device with a channel length of $0.2 \mu \mathrm{m}, \mathrm{n}^{+}$polysilicon top and bottom gates, a gate oxide of $70 \AA$, a thin film of $600 \AA$ doped with a p-type density of $4 \times 10^{17} \mathrm{~cm}^{-3}$, and various gate misalignments biased at $V_{G}=V_{\text {th }}$ and $V_{D}=50 \mathrm{mV}$, based on the analytical model with and without considering fringing electric field effect, and 2-D simulation results.

$V_{\text {th }}$ and $V_{D}=50 \mathrm{mV}$, based on the analytical model with and without considering the fringing electric field effect, and the 2-D simulation results.

As shown in the Fig. 3(a), when the gate misalignment increases, the back surface potential is lowered in the nongate overlap region. Considering the fringing electric field effect due to gate misalignment, the dip in the back surface potential becomes smaller as compared to the case without considering it. In addition, when the gate misalignment becomes larger, the fringing electric field effect becomes more important. As shown in Fig. 3(b), when the gate misalignment becomes larger, the fringing electric field effect on the front surface potential becomes more noticeable. In addition, a larger gate misalignment leads to a lower minimum front surface potential, which is also due to the fringing electric field effect. As shown in Fig. 3(a) and (b), the analytical model considering the fringing electric field effect could predict front and back surface potential distributions consistently as verified by the 2-D simulation results.

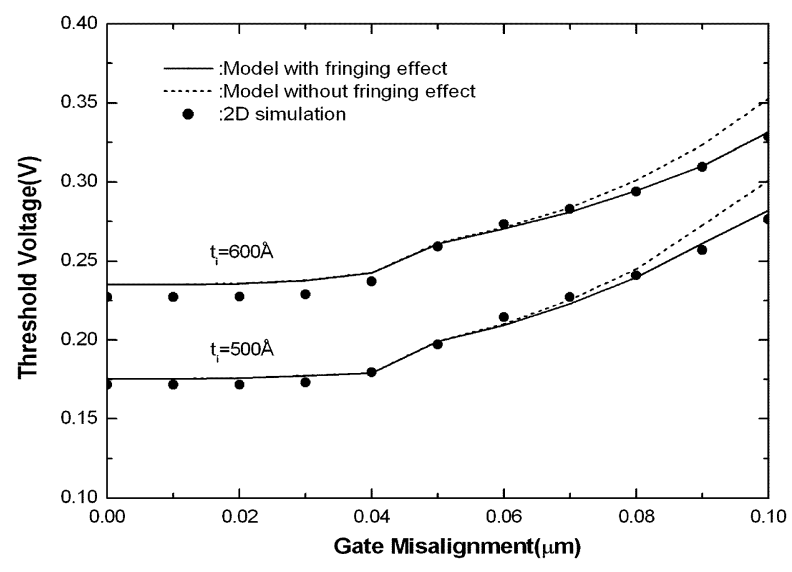

Fig. 4. Threshold voltage versus gate misalignment of the DG SOI nMOS device with a channel length of $0.2 \mu \mathrm{m}, \mathrm{n}^{+}$polysilicon top and bottom gates, a gate oxide of $70 \AA$, and a thin film of $500 \AA$ and $600 \AA$ doped with a p-type density of $4 \times 10^{17} \mathrm{~cm}^{-3}$, based on the analytical model with and without considering fringing electric field effect and 2-D simulation results.

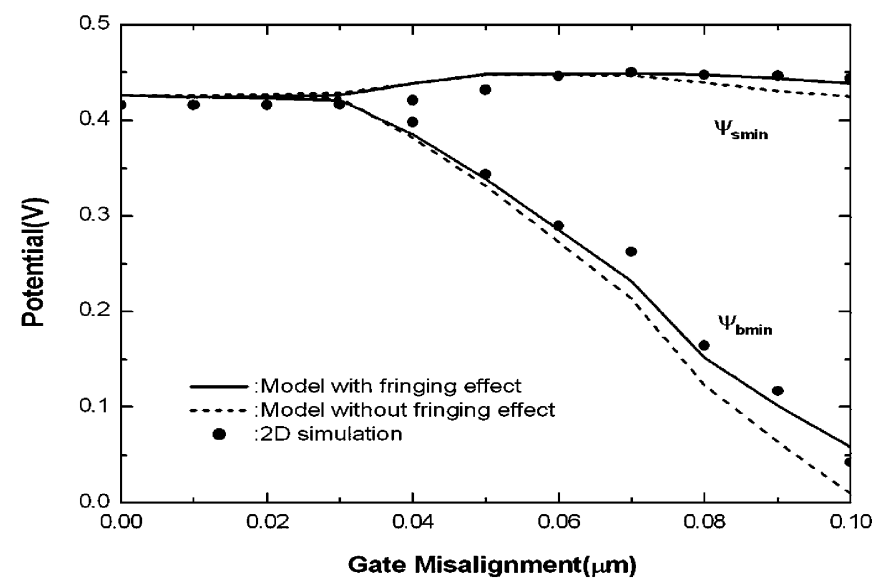

(a)

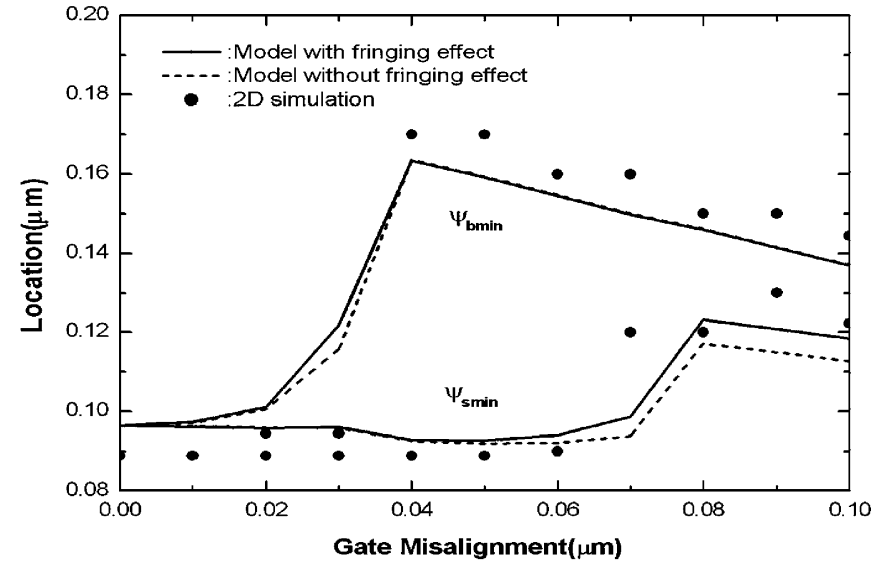

(b)

Fig. 5. (a) Minimum front and back surface potentials and (b) their locations versus gate misalignment of the DG SOI nMOS device with a channel length of $0.2 \mu \mathrm{m}, \mathrm{n}^{+}$polysilicon top and bottom gates, a gate oxide of $70 \AA$, and a thin film of $600 \AA$ doped with a p-type density of $4 \times 10^{17} \mathrm{~cm}^{-3}$, biased at $V_{G}=V_{\mathrm{th}}$ and $V_{D}=50 \mathrm{mV}$.

Fig. 4 shows the threshold voltage versus the gate misalignment of the DG SOI nMOS device with a channel length of $0.2 \mu \mathrm{m}, \mathrm{n}^{+}$polysilicon top and bottom gates, a gate oxide of 


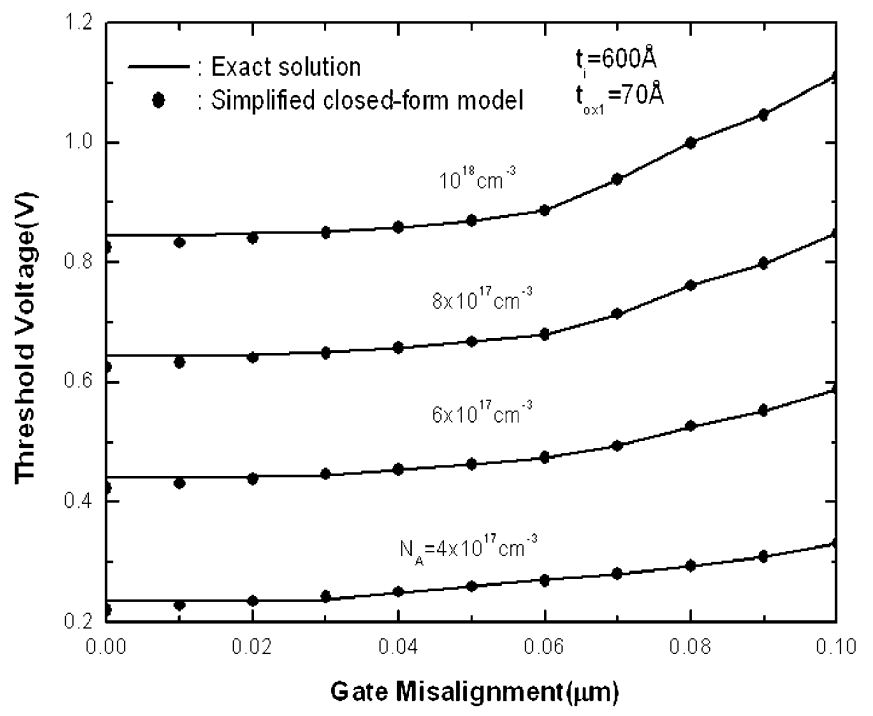

(a)

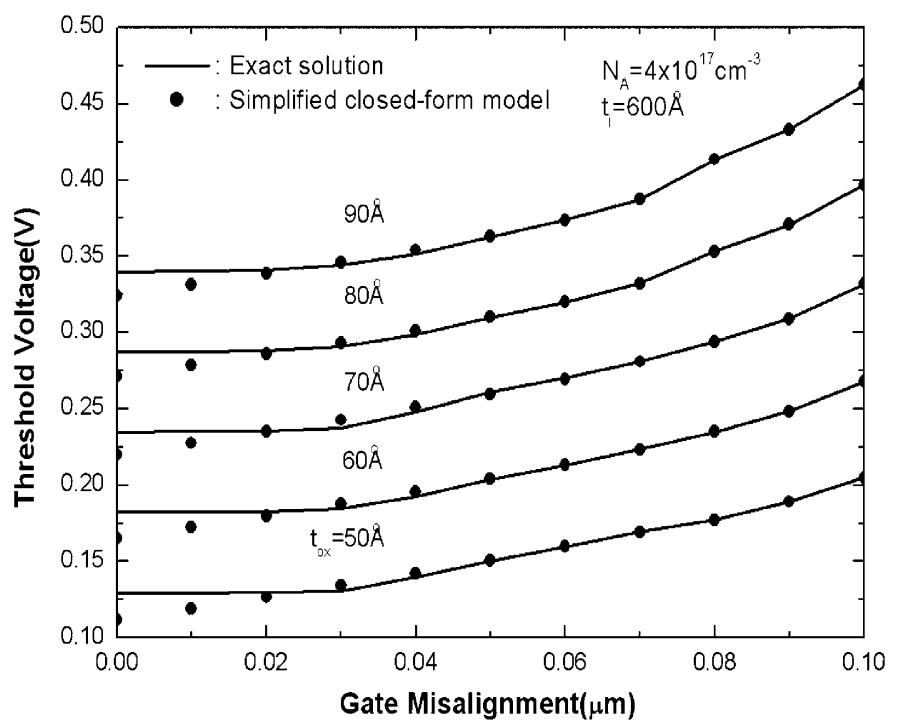

(c)

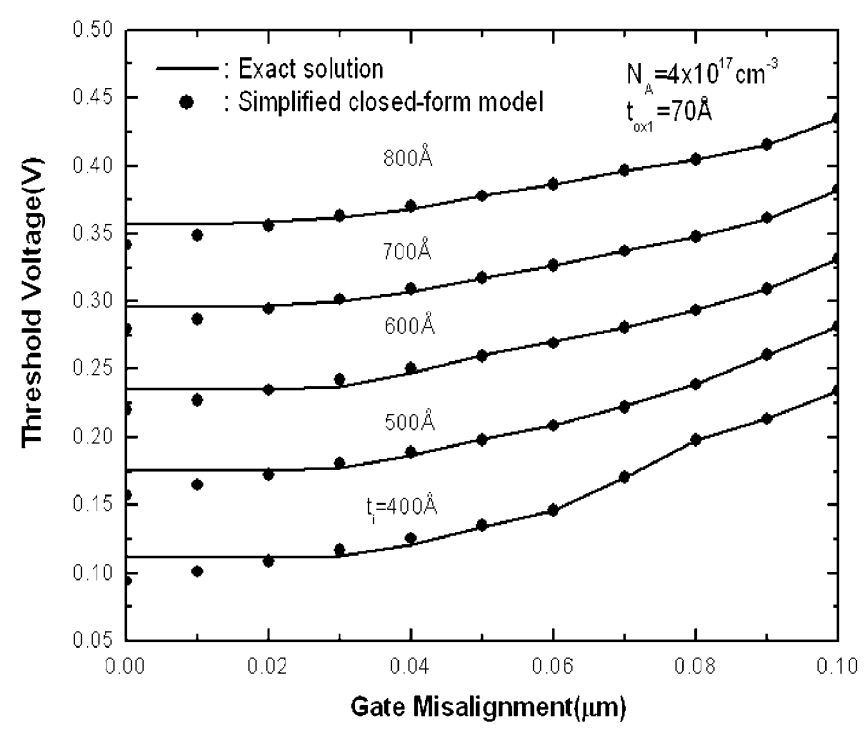

(b)

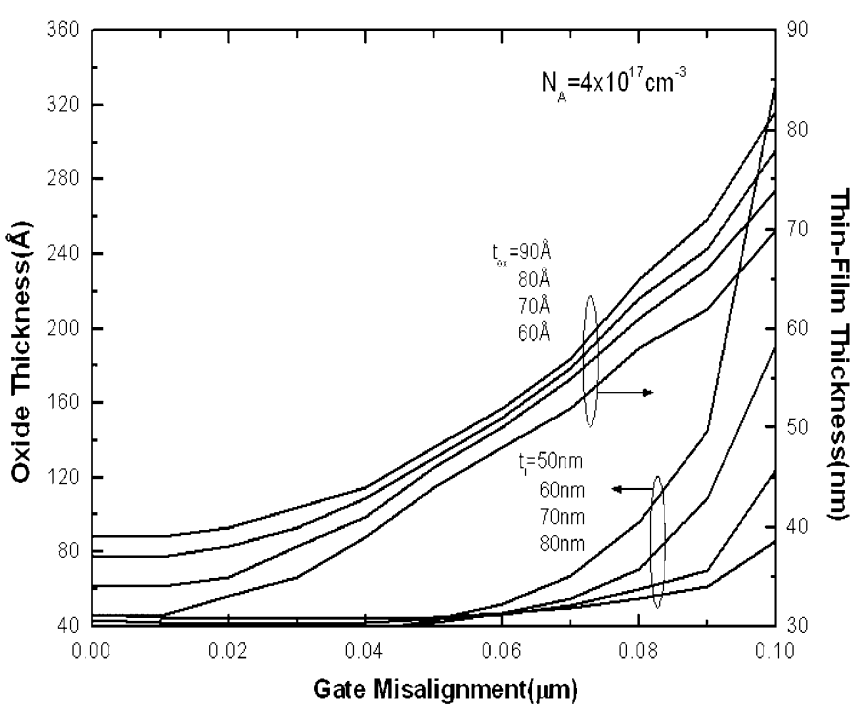

(d)

Fig. 6. Threshold voltage versus the gate misalignment of the DG SOI nMOS device with various (a) thin-film doping densities, (b) thin-film thicknesses, and (c) gate oxide thicknesses, using the exact solution from (30) and the simplified closed-form model [(31)-(40)]. (d) Criterion for selecting (31)-(36) or (37)-(40) for the threshold voltage.

$70 \AA$, and a thin film of 500 and $600 \AA$ doped with a p-type density of $4 \times 10^{17} \mathrm{~cm}^{-3}$, based on the analytical model with and without considering the fringing electric field effect and the 2-D simulation results. As shown in the figure, for both cases with a thin film of 500 and $600 \AA$, the fringing electric field effect coming from the gate misalignment is important in determining the threshold voltage. Without considering the fringing electric field effect, the threshold voltage is over-estimated, which is more noticeable when the gate misalignment becomes larger. At a gate misalignment of $0.1 \mu \mathrm{m}$, the fringing electric field effect causes a reduction in threshold voltage about $0.022 \mathrm{~V}(6 \%)$ for the thin film of $600 \AA$ and $0.028 \mathrm{~V}$ (7\%) for $500 \AA$. As shown in the figure, and verified by the 2-D simulation results, the analytical model considering the fringing electric field effect could provide an accurate prediction of the threshold voltage.

\section{DISCUSSION}

Fig. 5 shows (a) minimum front and back surface potentials and (b) their locations versus gate misalignment of the DG SOI nMOS device with a channel length of $0.2 \mu \mathrm{m}, \mathrm{n}^{+}$polysilicon top and bottom gates, a gate oxide of $70 \AA$, and a thin film of $600 \AA$ doped with a p-type density of $4 \times 10^{17} \mathrm{~cm}^{-3}$, biased at $V_{G}=V_{\text {th }}$ and $V_{D}=50 \mathrm{mV}$. As shown in Fig. 5(a) and (b), when the gate misalignment is smaller than $0.03 \mu \mathrm{m}$, the minimum front and back surface potentials are about equal, located in the gate overlap region. As the gate misalignment increases, the location of the minimum back surface potential moves toward the drain. At a gate misalignment of $0.04 \mu \mathrm{m}$, the minimum is located in the nongate overlap region. A further increase in the gate misalignment leads to a situation in which the 
location of the minimum back surface potential moves toward the source slightly. A similar situation exists for the front surface, but its transition point is located at a gate misalignment of $0.08 \mu \mathrm{m}$. When the gate misalignment is greater than $0.08 \mu \mathrm{m}$, the location of the minimum front surface potential moves into the nongate overlap region. As shown in the figure, as verified by the 2-D simulation results, the analytical model considering the fringing electric field effect predicts the behavior closely.

The closed-form analytical model developed in this paper is useful for providing insightful understanding while doing device design of DG SOI devices. Fig. 6 shows the threshold voltage versus the gate misalignment of the DG SOI nMOS device with various (a) thin-film doping densities, (b) thin-film thicknesses, and (c) gate oxide thicknesses, using the exact solution from (30) and the simplified closed-form model [(31)-(40)] and (d) the criterion for selecting (31)-(36) or (37)-(40) for the threshold voltage. As shown in Fig. 6(a)-(c), as the gate misalignment increases, the threshold voltage increases. When the thin-film doping density is raised, the threshold voltage becomes more sensitive to the gate misalignment [Fig. 6(a)]. At a doping density of $4 \times 10^{17} \mathrm{~cm}^{-3}$, with a gate misalignment of $0.1 \mu \mathrm{m}$, the threshold voltage is increased by $0.1 \mathrm{~V}$, while at a doping density of $10^{18} \mathrm{~cm}^{-3}$, it is increased by $0.27 \mathrm{~V}$. As shown in Fig. 6(b), when the thickness of the thin film is reduced, the threshold voltage becomes more sensitive to the gate misalignment. With a thin film of $800 \AA$, at a gate misalignment of $0.1 \mu \mathrm{m}$, its threshold voltage becomes larger by $0.077 \mathrm{~V}$, while with a thin film of $400 \AA$, it is increased by 0.124 V. As shown in Fig. 6(c), as the gate oxide thickness becomes smaller, the sensitivity of the gate misalignment effect becomes smaller. At a gate oxide of $90 \AA$, with a gate misalignment of $0.1 \mu \mathrm{m}$, the threshold voltage is increased by $0.123 \mathrm{~V}$, while at $50 \AA$, it is increased by $0.076 \mathrm{~V}$. Therefore, to minimize the gate misalignment effect, the thin film should not be too thin and its doping density should not be too high. In addition, the gate oxide thickness should be reduced to minimize the gate misalignment effect. From Fig. 6(a)-(c), as confirmed by the exact solution results using (30), the simplified closed-form model [(31)-(40)] could provide an accurate prediction of the gate misalignment effect. As described in the model derivation section, while deriving the threshold voltage, the minimum front surface potential in either the gate overlap $\left(\Psi_{s 1 \text { min }}\right)$ or the gate nonoverlap $\left(\Psi_{s 2 \min }\right)$ region has been used depending on its location. As described before, when

$$
\begin{aligned}
\left(\phi_{f n}+V_{D}\right. & \left.-D_{2}\right) \cosh \left(\sqrt{A_{0}} L_{m}\right)+\left[\phi_{f n}+V_{s}+D_{1}\right. \\
& \left.-\left(D_{1}+D_{2}\right) \cosh \left(\sqrt{A_{0}} L_{m}\right)\right] \sinh \left(\frac{\gamma}{k} m_{a}\right)<0
\end{aligned}
$$

which implies $g_{3}<g_{4}$, the minimum front surface potential occurs in the gate nonoverlap region $-\Psi_{s \min }=\Psi_{s 2 \text { min }}$ and (31)-(36) should be used. Otherwise, (37)-(40) should be used. The above equation is a function of the gate oxide thickness, and the thickness and the doping density of the thin film. Based on this reasoning, Fig. 6(d) shows the selection criterion for various gate oxide and thin-film thicknesses. As shown in the figure, for each case, to the right of the curve, $\Psi_{s 2 \text { min }}$ should be used in the threshold voltage formula [(31)-(36)] since the gate nonoverlap region dominates. To the left of the curve, $\Psi_{s 1 \text { min }}$ should be used since the gate overlap region is more important. When the gate oxide becomes thinner, the curve moves toward the right direction, which indicates that the possibility of the dominance of $\Psi_{s 2 \text { min }}$ in the gate nonoverlap region becomes smaller. When the thin film becomes thinner, the curve moves toward the left direction, which implies the increased chance of the dominance of $\Psi_{s 2 \mathrm{~min}}$.

\section{CONCLUSION}

In this paper, an analysis of the gate misalignment effect on the threshold voltage of DG FD SOI nMOS devices using a compact model considering the fringing electric field effect has been described. Using the conformal mapping transformation approach, a closed-form compact model considering the fringing electric field effect above the nongate overlap region has been derived, in order to provide an accurate prediction of the threshold voltage behavior, as verified by the 2-D simulation results.

\section{ACKNOWLEDGMENT}

The author would like to thank M. T. Lin of National Taiwan University for his kind help on simulation.

\section{REFERENCES}

[1] J. B. Kuo and S. C. Lin, Low-Voltage SOI CMOS VLSI Devices and Circuits. New York: Wiley, 2001.

[2] D. Hisamoto, "FD/DG-SOI MOSFET," in IEDM Tech. Dig., 2001, pp. 429-432.

[3] A. Vandooren, S. Cristoloveanu, and J. Colinge, "The dynamic conductance and transconductance in double-gate (Gate-all-around) SOI devices," in Proc. SOI Conf., Oct. 2000, pp. 116-117.

[4] K. Takeuchi, R. Koh, and T. Mogami, "A study of the threshold voltage variation for ultra-small bulk and SOI CMOS," IEEE Trans. Electron Devices, vol. 48, pp. 1995-2001, Sept. 2001.

[5] D. Esseni, M. Mastrapasqua, C. Fiegna, G. Celler, L. Selmi, and E. Sangiorgi, "An experimental study of low field electron mobility in double-gate, ultra-thin SOI MOSFETs," in IEDM Tech. Dig., 2001, pp. 445-448.

[6] B. Yu, T. Tanaga, and C. Hu, "Modeling off-state leakage current of DG-SOI MOSFET's for low-voltage design," in Proc. SOI Conf. , 1996, pp. $15-16$.

[7] H. Wong, K. Shin, and M. Chan, "The gate misalignment effects of the sub-threshold characteristics of sub-100 nm DG-MOSFETs," in Proc. HKEDM, 2002, pp. 91-94.

[8] P. Francis, A. Terao, D. Flandre, and F. V. de Wiele, "Modeling of ultrathin double-gate nMOS/SOI transistors," IEEE Trans. Electron Devices, vol. 41, pp. 715-720, May 1994.

[9] K. W. and J. B. Kuo, "A nonlocal impact ionization/lattice temperature model for VLSI double-gate ultrathin SOI nMOS devices," IEEE Trans. Electron Devices, vol. 44, pp. 324-330, Feb. 1997.

[10] S. Chen and J. Kuo, "Deep submicrometer double-gate fully-depleted SOI PMOS devices: A Concise short-channel effect threshold voltage model using a quasi-2-D approach," IEEE Trans. Electron Devices, vol. 43, pp. 1387-1393, Sept. 1996.

[11] T. Ushiki, M. C. Yu, Y. Hirano, H. Shimada, M. Morita, and T. Ohmi, "Reliable tantalum-gate fully-depleted-SOI MOSFET technology featuring low-temperature processing," IEEE Trans. Electron Devices, vol. 44, pp. 1467-1472, Sept. 1997.

[12] B. Maiti, P. J. Tobin, C. Hobbs, R. I. Hegde, F. Huang, D. L. O’Meara, D. Jovanovic, M. Mendicino, J. Chen, D. Connelly, O. Adetutu, J. Mogab, and J. B. La, "PVD TiN metal gate MOSFET's on bulk silicon and fully depleted silicon-on-insulator (FDSOI) substrates for deep sub-quarter micron CMOS technology," in IEDM Tech. Dig., 1998, pp. 781-784. 
[13] K. Uchida, J. Koga, R. Ohba, T. Numata, and S. Takagi, "Experimental evidences of quantum-mechanical effects on low-field mobility, gatechannel capacitance, and threshold voltage of ultrathin body SOI MOSFETs," in IEDM Tech. Dig., 2001, pp. 633-636.

[14] C. H. Choi, Z. Yu, and R. W. Dutton, "Two-dimensional polysilicon quantum-mechanical effects in double-gate SOI," in IEDM Tech. Dig., 2002, pp. 723-726.

[15] I. N. B. Senddon, Theme of Integral Transforms. New York: McGrawHill, 1972.

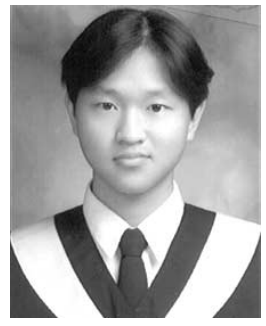

Elvis C. Sun was born in Taipei, Taiwan, R.O.C., on July 30,1978 . He received the B.S. degree in physics from National Central University, Chung-li, Taiwan, in 2000 and the B.S.E.E. and M.S.E.E. degrees from National Taiwan University, Taipei, in 2003.

$\mathrm{He}$ is currently serving his military obligation in the Taiwanese Defense Force. His research interest is in the compact modeling of SOI CMOS VLSI devices.

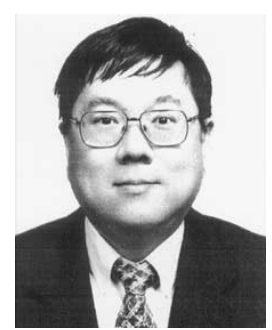

James B. Kuo (S'85-M'85-SM'92-F'00) received the B.S.E.E. degree from National Taiwan University (NTU), Taipei, Taiwan, R.O.C., in 1977, the M.S.E.E. degree from The Ohio State University, Columbus, in 1978, and the Ph.D.E.E. degree from Stanford University, Stanford, CA, in 1985.

He was with Penril Data Communications (1978-1981) as a Research Engineer working on integrating telecommunication modem chips using CMOS technology. From 1985 to 1987 , he was an Engineering Research Associate in the IC Lab of Stanford University, working on BiCMOS devices. In 1987, he joined NTU as an Associate Professor and, since 1990, he has been a Professor at the same university. In 2000, he joined the University of Waterloo, Waterloo, ON, Canada, as a tenured Full Professor, on leave from the NTU. In 2001, he was awarded the prestigious Canada Research Chair Professor by the Canadian government. His research expertise is in the field of low-voltage CMOS VLSI circuits and SPICE compact modeling of deep-submicrometer bulk and SOI CMOS and BiCMOS VLSI devices. As a leader of his research group at NTU, he has accomplished several breakthroughs in low-voltage CMOS digital circuits. In addition, his SPICE model for SOI CMOS technology, including fully depleted and partially depleted, is used among some of the top research institutions in the world. He has published 250 technical papers including 110 international journal papers, of which 50 are IEEE journal papers. He holds 16 invention patents including seven U.S. patents on low-voltage CMOS VLSI circuits. He has also authored nine books. As a highly recognized expert, he authored nine books including Low-Voltage SOI CMOS VLSI Devices and Circuits (New York: Wiley 2001), Low-Voltage CMOS VLSI Circuits (New York: Wiley 1999), and CMOS VLSI Engineering: Silicon-On-lnsulator (SOI) (Boston, MA: Kluwer 1998). As a technical leader, he has graduated 50 M.S. and Ph.D. students who specialized in CMOS circuit designs and device modeling and are currently working in leading U.S. and Taiwan microelectronics companies.

Dr. Kuo is an IEEE Fellow for contributions to modeling CMOS VLSI devices. He serves as an international advisory committee member for the IEEE CIRCUITS AND DEVICES MAGAZINE and the membership committee chair for the IEEE Electron Devices Society. He is also an IEEE Electron Devices Society Distinguished Lecturer. 